\title{
Desenvolvimento da Bota de Tração na Impressora 3D
}

\author{
Boot of Traction Development on 3D Printer
}

\author{
Desarollo de La Bota de Tracción en la Impresora 3D
}

\section{Gisele Roque de Souza ${ }^{1 *}$, Tiana Carneiro Simões de Almeida ${ }^{2}$, Matheus da Silveira Fagundes Rodrigues $^{3}$, Norival Garcia da Silva $\mathbf{J r}^{4}$, Adauri Silveira Rodrigues $\mathbf{J r}^{5}$, Eduardo Tavares Lima Trajano $^{6}$}

Como citar esse artigo. de Souza, GR; de Almeida, TCS; Rodrigues, MSF; Rodrigues Jr, N.G.S; Jr, AS; Trajano, ETL. Desenvolvimento da Bota de Tração na Impressora 3D. Revista PróUniverSUS. 2021 Jan./Jun.; 12 (1): 100108 .

\section{Resumo}

O tratamento inicial das fraturas femorais é feito com a tração do membro inferior com fixação transcutânea ou transesquelética. Ambas as técnicas possuem chance de complicação. As patentes são recursos de investimento em pesquisa aplicados por uma empresa, assegurado por lei e depositadas no INPI. Material e Método: Foi utilizado o SolidWorks® 2013 para desenvolvimento da bota em formato anatômico utilizando medidas adquiridas de uma perna adulta. Resultado: A Bota de Tração é composta por uma bota anatômica, forrada de espuma, apoiada sob um suporte de aço onde é feita a tração através de motor de passo ou manualmente e demonstrada em display digital. Trata-se de uma inovação por proteger a pele e o osso da tração e além disso, permitir mensurar exatamente a quantidade a ser aplicada. Discussão: Uma busca mundial no banco de patentes analisou cinco produtos com diferentes graus de similares usados para tracionar o membro inferior, entretanto nenhum tinha a finalidade proposta pela Bota de Tração, corroborando sua inventividade. Conclusão: Sendo um produto inovador no mundo, foi realizado um pedido para o depósito no INPI e gerado o número de registro do processo.

Palavras-chave: Fraturas do Fêmur; Tração; Patente.

\begin{abstract}
Introduction: The initial treatment of femoral fractures are done with inferior limb traction with transcutaneous or transkeleton fixation. Both techniques have a chance of complications. Patents are investiment resources applied on research by a company, assured by law and deposit in INPI. Materials \& Methods: The software SolidWorks ${ }^{\circledR} 2013$ was used for the development of the boot in anatomic shape using measures of an adult leg. Results: The Traction Boot has an anatomic boot with foam on the inside over a still support where the traction is made with a stepper motor or manually and demonstrated on a digital display. It's an innovation because it protects the skin and the bones from a direct traction force besides mesuring the exact amount of force to be made. Discussion: A world research on the patents bank found five similar products used to traction the inferior limb with different degrees of similarity, however none of them had the proposition of the Boot of Traction, what makes it innovative. Conclusion: Being an innovative product around the world, a patent request was placed for a deposit on INPI and a register number of the process was generated.
\end{abstract}

Keywords: Femoral Fractures; Traction; Patent.

\footnotetext{
Afiliação dos autores:

${ }^{1}$ Discente Graduação de Medicina da Universidade de Vassouras, Vassouras, Rio de Janeiro, Brasil. ORCID: https://orcid.org/0000-0003-4450-8052

${ }^{2}$ Discente Graduação de Medicina da Universidade de Vassouras, Vassouras, Rio de Janeiro, Brasil. ORCID: https://orcid.org/0000-0001-7001-8933

${ }^{3}$ Discente do $3^{\circ}$ ano do Ensino Médio do Colégio Estadual Ministro Raul Fernandes, Vassouras, Rio de Janeiro, Brasil. ORCID: https://orcid.org/0000-0001-9168-4644

${ }^{4}$ Discente do Mestrado de Ciências Médicas da Universidade de Vassouras, Vassouras, Rio de Janeiro, Brasil. ORCID: https://orcid.org/0000-0001-8424-7286

${ }^{5}$ Doutor. Docente do Mestrado de Ciências Médica da Universidade de Vassouras, Vassouras, Rio de Janeiro, Brasil. ORCID: https://orcid.org/0000-0001-8982-0221

${ }^{6}$ Doutor. Docente do Mestrado de Ciências Médicas da Universidade de Vassouras, Vassouras, Rio de Janeiro, Brasil. ORCID: https://orcid.org/0000-0001-7809-7138
} 


\section{Resumen}

Introducción: el tratamiento inicial de las fracturas femorales se realiza mediante tracción del miembro inferior con fijación transcutánea o transesquelética. Ambas técnicas tienen posibilidades de complicarse. Las patentes son recursos de inversión en investigación aplicados por una empresa, garantizados por ley y depositados en el INPI. Material y método: Se utilizó SolidWorks ${ }^{\circledR} 2013$ para desarrollar la bota en forma anatómica utilizando medidas adquiridas de una pierna adulta. Resultado: La Bota de Tracción está compuesta por una bota anatómica, forrada con espuma, apoyada bajo un soporte de acero donde la tracción se realiza mediante un motor paso a paso o manualmente y se demuestra en una pantalla digital. Es una innovación para proteger la piel y el hueso de la tracción y, además, permite medir exactamente la cantidad a aplicar. Discusión: Una búsqueda mundial en el banco de patentes analizó cinco productos similares utilizados para tirar del miembro inferior, sin embargo ninguno tenía el propósito propuesto por Traction Boot, corroborando su inventiva. Conclusión: Siendo un producto innovador en el mundo, se realizó un pedido para el depósito en el INPI y se generó el número de registro del proceso.

Palabras clave: Fracturas del Fémur; Tracción; Patente.

\section{Introdução}

Fraturas femorais diafisárias são aquelas que ocorrem no corpo do fêmur e tem como característica serem graves e se originarem de traumas de grande energia com alta dissipação de energia cinética tais como acidentes automobilísticos ${ }^{1-2}$.

A classificação de Winquist é usada avaliar e classificar a fratura segundo o grau de cominuição e é dividida em quatro tipos, sendo a tipo I de cominuição simples ou mínima, tipo II àquela com cominuição de até $50 \%$ do diâmetro da diáfise, tipo III com cominuição de 50 a $100 \%$ e tipo IV cominuição completa sem contato entre as extremidades da fratura após a redução².

A técnica de tração é usada para tratamento de fraturas femorais que têm como local de acometimento a diáfise do osso ${ }^{3}$. A tração é feita como um tratamento pré-cirúrgico afim de aliviar a dor do paciente enquanto este aguarda o procedimento de fixação definitivo e consiste em uma fixação distal na região proximal tibial ou no calcâneo ${ }^{3}$ com a força aplicada através de um peso preso na extremidade do membro inferior (Figura 1).
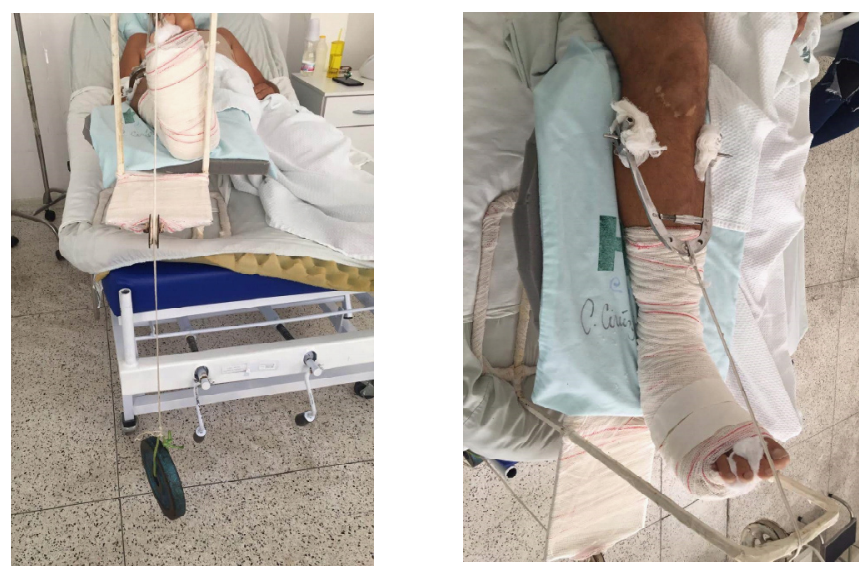

Figura 1. Fixação transesquelética em tíbia proximal com tração usando uma anilha.

Fonte: Cedida pelo Dr. Norival Garcia da Silva Jr (2019)
Tradicionalmente, usa-se a via transcutânea ou a transesquelética através de pinos para a realização desta técnica com a aplicação de pesos equivalentes à $10 \%$ do peso do paciente. No adulto, a fixação transesquelética é feita mais frequentemente e apresenta uma série de possíveis riscos como infecção através dos pinos causando artrite séptica no joelho quando fixada na tíbia proximal, assim como possibilidade de lesar o nervo poplíteo e a artéria poplítea causando um pseudoaneurisma ${ }^{4}$.

Visando sanar os problemas referentes à fixação para realizar a força de tração necessária no préoperatório, a fim de melhorar o prognóstico do paciente com fratura femoral, é que foi desenvolvida a Bota de Tração. $\mathrm{O}$ formato anatômico da bota somado ao material em espuma do interior cria uma cobertura integral da região tibial e calcânea com proteção à força aplicada pela tração. Esta é feita manualmente ou por motor de passo e registrada via display digital que permite controlar exatamente o montante de força aplicada.

As patentes são recursos gerados por investimento em pesquisa e desenvolvimento que uma empresa aplica no desenvolvimento de inovações. Ao serem geradas ficam sob um depósito de patentes ficam asseguradas por lei e em troca fornece seus dados detalhados do conteúdo técnico que a inovação se trata.

Ao depositar uma patente, esta é armazenada e disponibilizada no Banco de Patentes do Instituto Nacional de Propriedade Industrial (INPI). Para os inventores detentores de uma patente é vantajoso o depósito, uma vez que permite remunerar sua pesquisa científica e o desenvolvimento tecnológico feito e, além disso, contribui para o enriquecimento nos âmbitos científicos, econômicos e do desenvolvimento tecnológico de todo o país ${ }^{5}$.

Objetivo desenvolver dispositivo de tração de membros inferiores com bota ortopédica de formato anatômico. 


\section{Descrição do Produto}

Foi desenvolvido um dispositivo de tração de membros inferiores com bota ortopédica de formato anatômico e controle de cara digital que permite a tração da articulação coxofemoral daqueles acometidos por fratura de fêmur. $\mathrm{O}$ desenho foi desenvolvido via software SolidWorks ${ }^{\circledR} 2013$ usando medidas adquiridas da anatomia de uma perna adulta.

Em virtude de se tratar de uma invenção com soluções inovadoras à técnica de tração de membros inferiores para tratamento da fratura de fêmur, a Bota de Tração teve um depósito requerido de patente junto ao seu desenvolvimento com o número de depósito BR 1020200149180.

O produto Bota de Tração é composto por uma bota em formato anatômico feita de plástico (Figura 2) e um suporte feito de aço inoxidável ou 1020 (Figura 3).

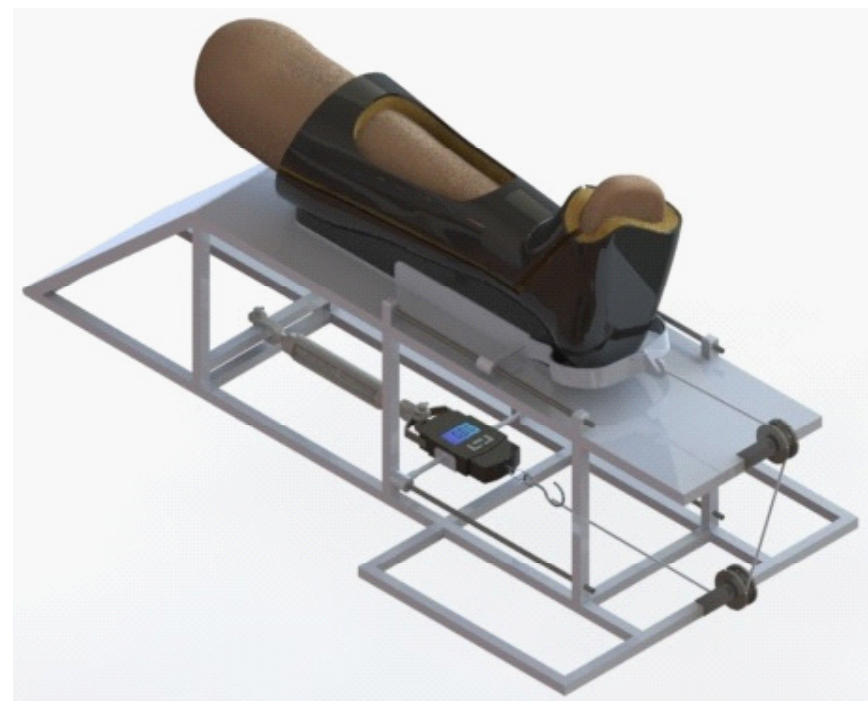

Figura 2. Bota de Tração.

Fonte: Autores (2020).

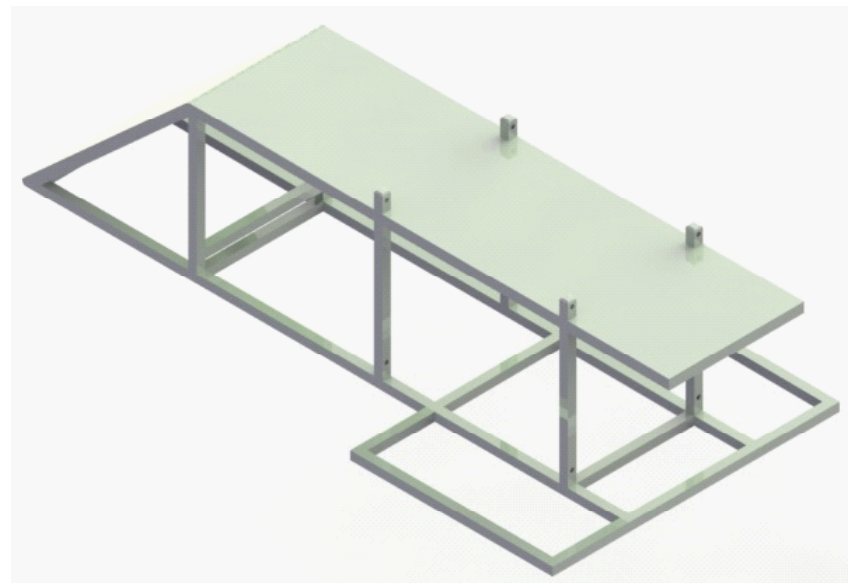

Figura 3. Suporte de Aço Inox 1020.

Fonte: Autores (2020).
Os componentes - capa de roldana, haste guia, rolamento linear, suporte do rolamento linear, pino de roldana, roldana, cabo de aço, suporte da balança, balança, presilha do esticador, pino do esticador, rosca com M10 do esticador e corpo do esticador constam no suporte. A bota possui uma capa inferior, uma espuma inferior, espuma superior e capa superior plástica. Estas se juntam e se unem a pena do paciente com fixadores em velcro ou correia com fivela em couro resistente (Figura 4).

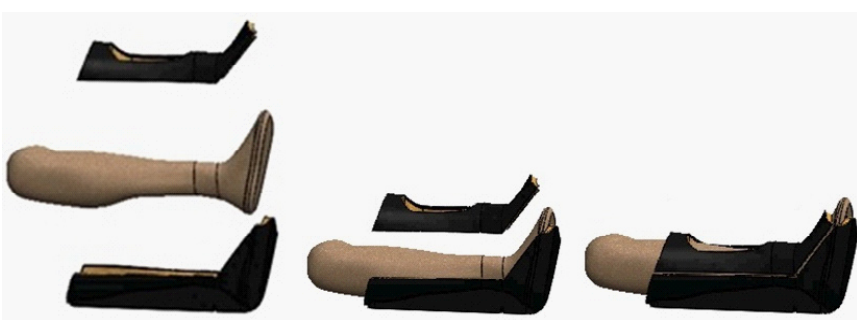

Figura 4. Detalhes da Bota.

Fonte: Autores (2020).

Ao desenvolver o produto Bota de Tração, a fim de ser feito o pedido de depósito de patente, foi feita uma busca mundial em bancos de patentes e foram encontradas cinco patentes com a mesma depositadas que variam entre baixa, média ou alta similaridade conforme a semelhança ao produto da Bota de Tração.

A patente brasileira 202016018267-6U2 descreve um dispositivo para estabilizar membros inferiores durante procedimentos cirúrgicos usando tração ${ }^{6}$. Ainda que não usado no mesmo fim que a Bota de Tração, usada para tração pré-operatória, o produto da patente brasileira se mostraria com problemas como um apoio único na região calcânea que poderia em um uso prolongado provocar úlcera de pressão. Isto permite também que haja rotação do membro, podendo gerar instabilidade e prejudicar seu bom uso.

A patente brasileira não possibilita a mensuração da força aplicada, pois não tem nenhum equipamento de demonstração e mensuração. Além disso, permanece o uso da fixação transesquelética ou transcutânea, permanecendo os problemas dessas técnicas.

Na patente norte-americana depositada no número US9949861 B2 prevê um produto de suporte usado no ambiente intraoperatório que permite a manipulação da perna sem que haja perda da tração durante a cirurgia ${ }^{7}$. Sua fixação é feita através de uma bota. Apesar de seu uso na aplicação da tração ser em momento diferente do dispositivo da Bota de Tração, o uso prolongado da bota desenvolvida pela patente norte-americana poderia levar à úlcera de pressão da região calcânea, uma vez 
que esta bota não contempla integralmente o membro inferior deixando esta região exposta.

Além disso, a ausência de um suporte que fixe a bota pode levar a movimentação do membro prejudicando o tratamento da imobilização e tração. No depósito de patente norte-americano US 5582379A trata-se de um dispositivo que se acopla a mesa cirúrgica com uma haste que pode suportar o membro inferior através de um estribo ou uma bota ${ }^{8}$.

Sua aplicabilidade é em procedimentos cirúrgicos. Ainda que não usado para o fim pré-operatório, nesta patente o produto apresenta pouco suporte para o membro inferior, recaindo novamente no problema se possibilidade de rotação do membro que seria possível já que o paciente não estaria anestesiado e sedado como no momento cirúrgico. $\mathrm{O}$ apoio suporte desenvolvido não consta com nenhuma proteção para úlceras de pressão e além do mais, o sistema não conta com um meio mensurável preciso de aplicação da força de tração.

A patente US 4653482 norte-americana também descreve um produto para ser usado no intra-operatório ${ }^{9}$, porém além dos problemas de fixação do membro a ser tracionado, possibilidade de úlcera de pressão em uso prolongado e ausência de possibilidade de mensurar precisamente a força aplicada, este produto apresenta um suporte que permite que seja usado para tracionar o membro superior pois serve de apoio para a região da fossa cubital $^{9}$. Entretanto este mesmo suporte impede a centralização correta do membro inferior com o paciente em decúbito dorsal, logo o paciente fica lateralizado o que pode promover ainda mais regiões de úlcera de pressão como na região do ísquio púbico e no cotovelo e, somado a falta de estabilização na fixação do membro tracionado, prejudicar a eficácia do tratamento com a tração.

A similaridade das patentes supracitadas com a Bota de Tração foi baseada no uso da tração, mas por não se tratar do mesmo fim terapêutico do dispositivo aqui desenvolvido, foi considerada nas buscas uma relação de baixa relevância na defesa do pedido de depósito da patente. Na busca por similaridade apenas uma patente tinha a mesma proposta terapêutica pré-operatória do nosso dispositivo, sendo esta a patente norte-americana de número US 7,052,479 B2 que propõe a aplicação da tração através de um estribo fixada em uma espuma que recobre a parte posterior do membro inferior e com duas faixas na região anterior situadas mais proximal e outra distalmente na região tibial. Para aplicação da tração sugere o uso de pesos de $4,5 \mathrm{~kg}$ equivalentes a aproximadamente 10 pounds presos a uma corda que passa por uma roldana e o peso na extremidade pendente.

Este dispositivo, então, apresenta solução para o calcanhar apoiado, prevenindo úlcera de pressão e um sistema de mensuração por pesos ${ }^{10}$. Entretanto, não há uma proteção integral da pele do membro tracionado, deixando a região anterior exposta e ainda com faixas de fixação que podem levar a lesão da pele daquela área de forma semelhante ao que ocorre na tração transcutânea. $\mathrm{O}$ fato da força ser aplicada sob uma estrutura de espuma torna o produto após o uso mais desgastado, pois a resistência das fibras da espuma é baixa e cedem conforme o tempo de uso, tornando o produto altamente perecível.

Diante desta busca por depósitos de patente, foi visto que a Bota de Tração oferece soluções superiores aos produtos patenteados, pois o formato anatômico da bota se ajusta ao paciente e protege integralmente as regiões posterior e anterior da tração. Por ser composta de plástico, apresenta uma resistência maior para resistir à aplicação de tração e, portanto, maior durabilidade do produto. $\mathrm{O}$ revestimento interno de espuma permite a proteção contra úlcera de pressão do calcanhar e também de toda a pele e, por ser removível, pode ser substituída quando reutilizado o sistema da Bota de Tração, tornando ainda mais seguro seu uso em outros pacientes bastando trocar as espumas internas.

No suporte, a bota é encaixada através de um encaixe sob um entalhe na capa inferior da bota (Figura $5)$.

Trata-se de uma inovação por sua fixação através da bota com interior acolchoado e ajustável ao tamanho do paciente dispensar acesso cirúrgico ou aplicação de tração diretamente à pele ou ao osso e permitir que com o motor de passo ou manualmente esta força seja mensurada e demonstrada em display digital, otimizando

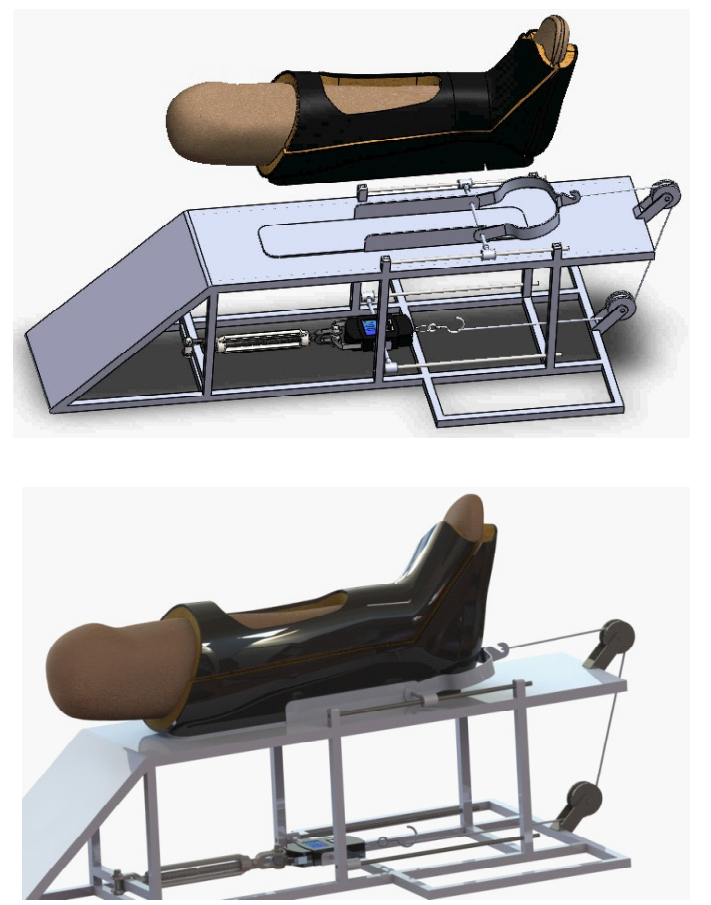

Figura 5. Detalhes do encaixe da Bota no esticador para que seja aplicada a força de tração..

Fonte: Autores (2020). 
o tratamento da fratura femoral do paciente.

\section{Implicações para a Prática}

Quanto a aplicabilidade, o produto oferece um suporte de apoio integral do membro inferior em tratamento que por possuir um encaixe para a bota, consegue ainda a estabilização deste, melhorando o posicionamento da fratura e consequentemente $o$ prognóstico do paciente.

\section{Considerações Finais}

A Bota de Tração é um produto que possuí caráter inovador que soluciona os problemas das técnicas tradicionais para tratamento da fratura de fêmur e articulação coxofemoral. Por possuir uma bota anatômica, com revestimento interno com espuma promove proteção integral ao membro tracionado tanto de infecções e lesões vasculares por dispensar acesso cirúrgico para fixação de pinos tanto da pele por não aplicar a força de tração diretamente so a pele.

O sistema de balança com display digital permite exatidão na força aplicada que pode ser feita tanto via manual quanto motor de passo. Sendo assim, o produto desenvolvido pelo Mestrado de Ciências Médicas da Universidade de Vassouras é superior àqueles encontrados atualmente no mercado, justificando seu depósito em patente.

\section{Referências}

1. Rosseti Antônio Carlos, Ricco Jr Laércio Francisco, Moraes Maurício de, et al. Tratamento das fraturas complexas da diáfise femoral com "interlocking nail". Rev Bras de Ortop [Internet]. 1997 [cited 2021 Jan 26];32 Available from: http://rbo.org.br/detalhes/556/pt-BR/tratamento-dasfraturas-complexas-da-diafise-femoral-com--interlocking-nail-

2. Pires Robinson Esteves Santos, Reis Fernando Baldy dos, Simões Christiano Esteves, Santos Leandro Emílio Nascimento, Rodrigues Vinícius Bicalho, Andrade Marco Antônio Percope de et al . Fratura diafisária do fêmur: reprodutibilidade das classificações AO-ASIF e Winquist. Acta ortop. bras. [Internet]. 2010 [cited 2021 Jan 26] ; 18( 4 ): 197-199. Available from: $\quad$ http://www.scielo.br/scielo.php?script=sci arttext\&pid=S141378522010000400004\&lng=en. http://dx.doi.org/10.1590/S141378522010000400004

3. Belangeo William Dias, Fernandes Paulo Magalhães, Inamine Jorge Thoei. Fraturas diafisárias do fêmur: estudo comparativo entre os métodos de Küntscher e de Ender*. Rev Bras Ortop [Internet]. 1994 [cited 2021 Jan 26];29 Available from: https://www.rbo.org.br/detalhes/808/pt-BR

4. Stefl MD, Azad A, Antonios JK, Carney J, Marecek GS. Safety of skeletal traction through the distal femur, proximal tibia, and calcaneus. Arch Trauma Res [serial online] 2019 [cited 2021 Jan 26];8:198-202. Available from: https://www.archtrauma.com/text.asp?2019/8/4/198/272840

5. Ferreira Ademir Antônio, Guimarães Edilson Rodrigues, Contador José Celso. Patente como instrumento competitivo e como fonte de informação tecnológica. Gest. Prod. São Carlos [Internet]. 2009 [cited 2021 Jan 26];16:209 - 221. Available from: https:/www.scielo.br/pdf/gp/v16n2/ v16n2a05.pdf

6. Lima Diego Ariel de, Lima Luiz Lopes, inventors; Dominos Luis
André Santos, assignee. Dispositivo de Tração Ortopédica Portátil. BR 202016018267-6 U2. 2018 Feb 27.

7. Drennan Denis Burke, inventor; Hartman Gary M.; Hartman Domenica N. S.; Hartman \& Hartman, assignee. Traction Device. US9949861 B2. 2006 Apr 20.

8. Kreuzer Stefan, Menzi Manfred, Stark Kurt, inventors; Rose Conley, assignee. Devices and methods for guiding and applyin traction to a patients leg during surgery. US 2015/0190265 Al. 2019 Jul 9.

9. Keselman Yury, Skinner George E., Navarro Richard R., inventors; Gordon, McCoy \& Granger, assignee. Adjustable limb support system. US 5,582,379. 1995 May 28 .

10. Kurtland Kenneth Z., inventor; Charmasson \& Holz, assignee. UpperExtremity traction tray attachment for operating table. US 4,653,482. 1985 May 17. 


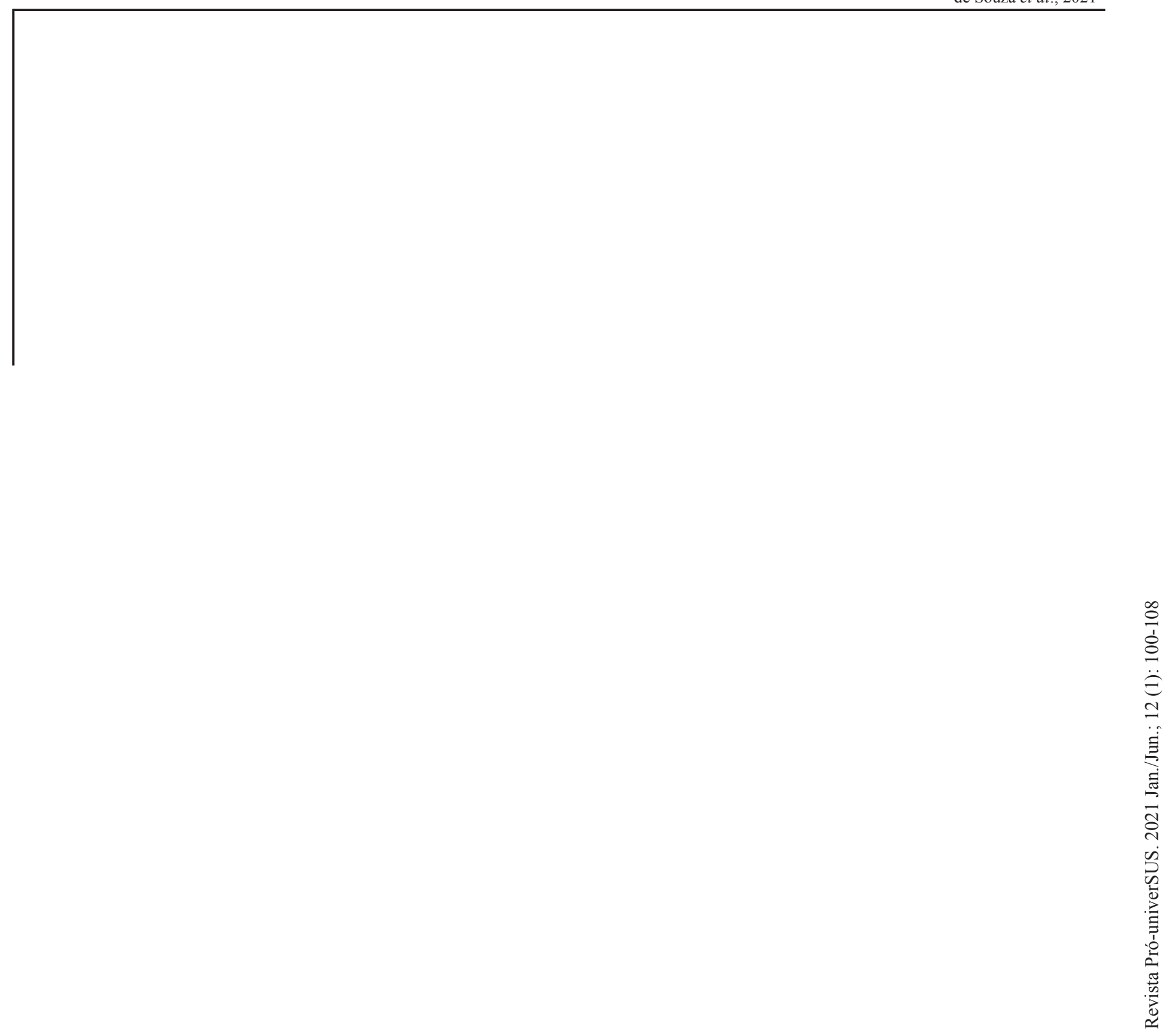


de Souza et al., 2021. 


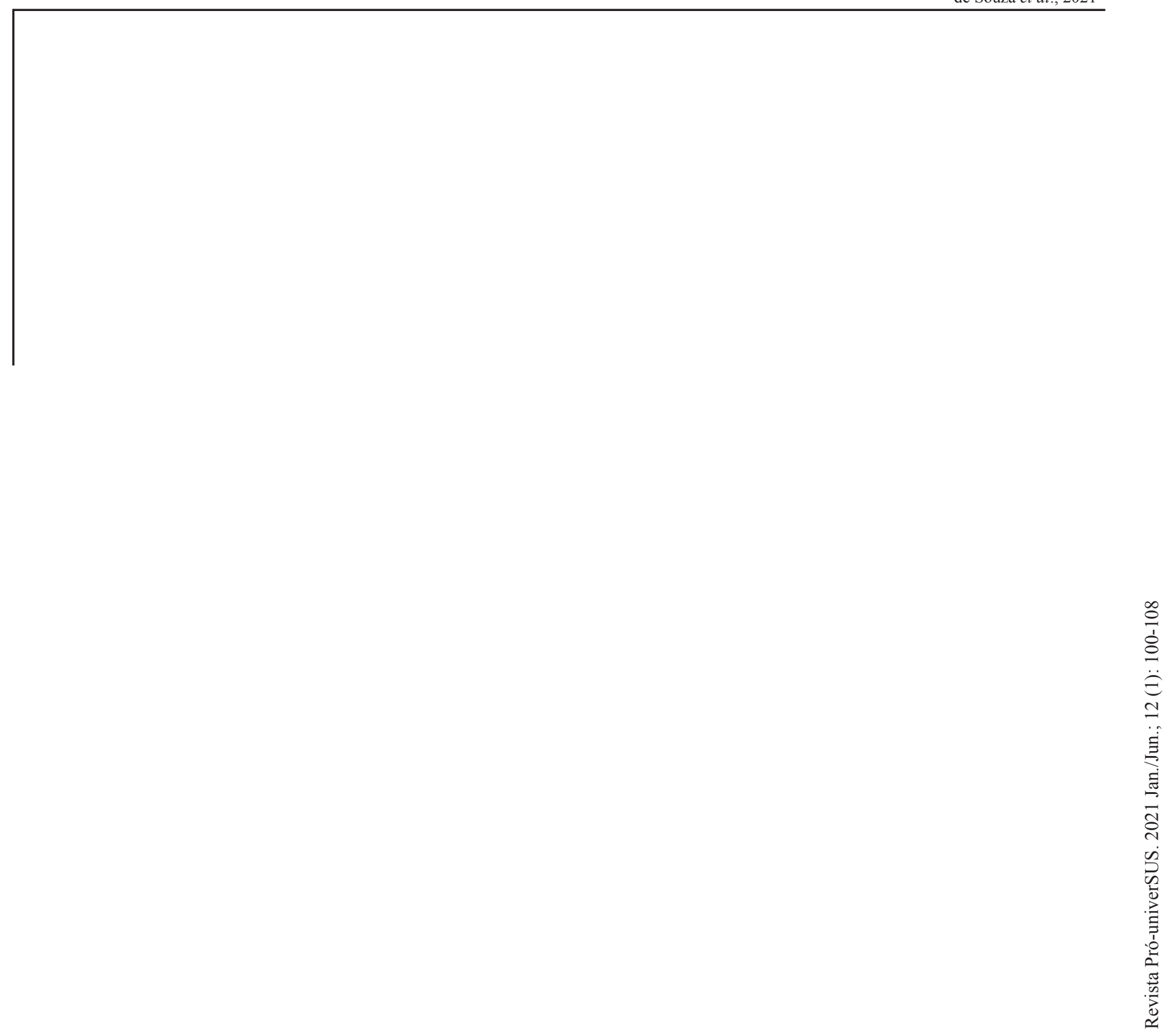

